\title{
Loss of large myelinated nerve fibres of the recurrent laryngeal nerve in patients with multiple system atrophy and vocal cord palsy
}

\author{
M Hayashi, E Isozaki, M Oda, $\mathrm{H}$ Tanabe, J Kimura
}

Department of

Neurology

M Hayashi

E Isozaki

H Tanabe

Department of

Neuropathology,

Tokyo Metropolitan

Neurological Hospital,

Tokyo, Japan

M Oda

Department of

Neurology, Faculty of

Medicine, Kyoto

University, Kyoto,

Japan

J Kimura

Correspondence to:

Dr M Hayashi, Department of Neurology, Tokyo

Metropolitan Neurological

Metropolitan Neurological

Fuchu City, Tokyo, 183, Japan.

Received 10 May 1996 and in revised form 17 September 1996

Accepted 1 November 1996

\begin{abstract}
Objectives-Vocal cord palsy seen in some patients with multiple system atrophy may result from neuronopathy of the recurrent laryngeal nerve.

Methods-Six controls and six patients with multiple system atrophy, four with and two without vocal cord palsy were studied. The number of myelinated nerve fibres were counted and fibre diameter histograms were established for the motor and sensory divisions of the laryngeal branch of the recurrent laryngeal nerve.
\end{abstract}

Results-Although both groups of patients with multiple system atrophy showed selective loss of the myelinated fibres in the motor branch, the change was greater in those with vocal cord palsy than in those without. The small myelinated nerve fibres (diameter $<7 \mu \mathrm{m}$ ) were decreased in number in both multiple system atrophy groups, whereas the large myelinated nerve fibres (diameter $<8 \mu \mathrm{m}$ ) were decreased only in those with vocal cord palsy, and preserved in those without the symptom.

Conclusion-In multiple system atrophy, the small myelinated fibres innervating the vocal cord are affected first, without obvious clinical signs. The patient develops vocal cord palsy only after the loss of the large myelinated fibres, which mostly comprise the $\alpha$ motor axons that innervate the intrinsic laryngeal muscles.

$(\Im$ Neurol Neurosurg Psychiatry 1997;62:234-238)

Keywords: recurrent laryngeal nerve; multiple system atrophy; vocal cord palsy

Vocal cord palsy is common and may become life threatening in multiple system atrophy. ${ }^{123}$ Histological and EMG reports on multiple system atrophy have documented neurogenic changes in the posterior cricoarytenoid muscles, the sole abductor of the vocal cords. ${ }^{24}$ Bannister et al, in the only pathological study of the recurrent laryngeal nerve in multiple system atrophy, found no gross abnormalities in one patient and no focal changes in another. ${ }^{2}$ They made no attempt to document the number of myelinated fibres, the fibre diameter distribution, or the difference between the motor and sensory branches. The recurrent laryngeal nerve divides into the cricopharyngeal branch, which innervates the cricopharyngeal and inferior constrictor muscles, and the laryngeal branch, which sub-

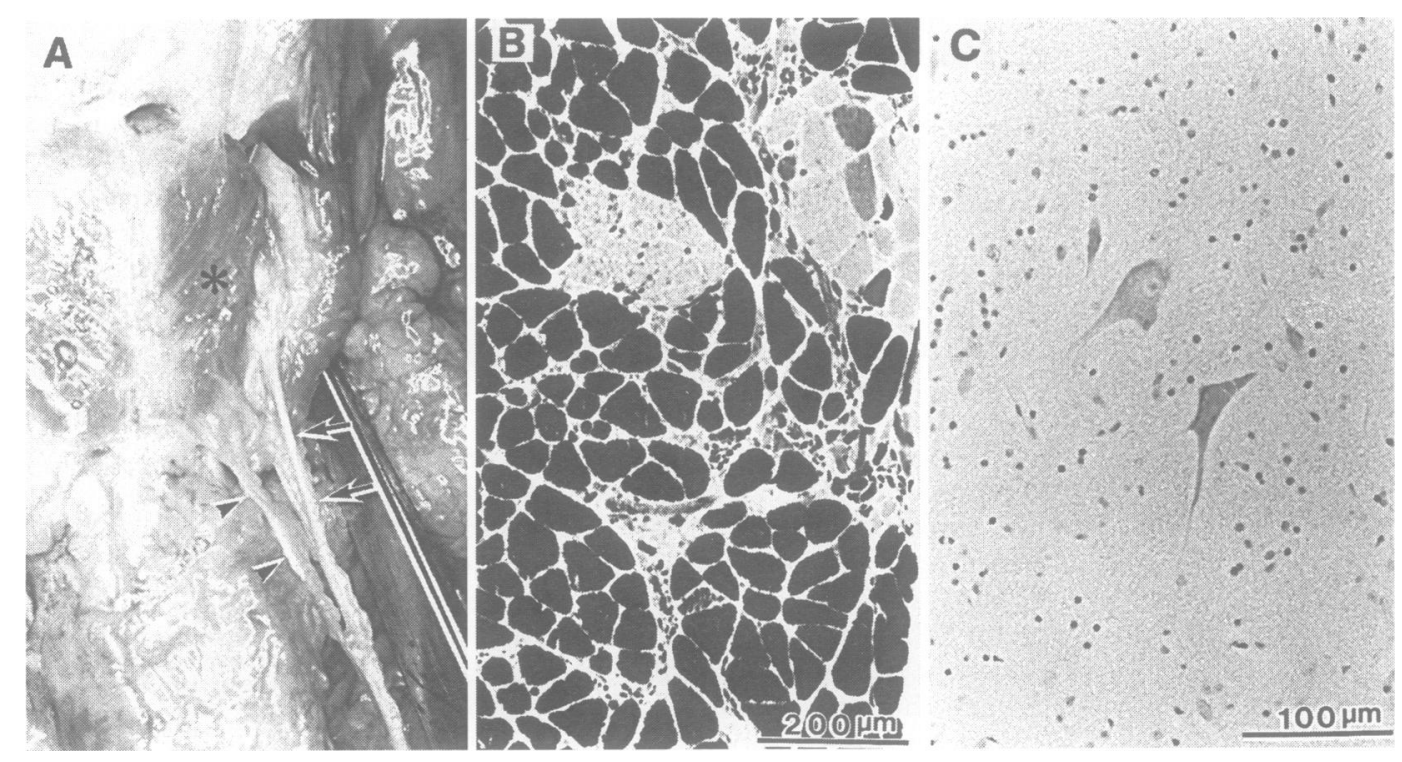

Figure 1 (A) Posterior view of the laryngeal branch of the recurrent laryngeal nerve abutting the posterior cricoarytenoid muscle $\left(^{*}\right)$. The laryngeal branch divides into the thicker anterior motor (arrow) and thinner posterior sensory

(arrowhead) divisions. The anterior motor division ascends lateral to the posterior cricoarytenoid muscle after sending small branches to innervate this muscle. (B) The posterior cricoarytenoid muscle from a patient with multiple system atrophy and vocal cord palsy (stained with ATPase), showing grouped atrophy and fibre type grouping. (C) The nucleus ambiguus stained with Nissl stain, showing gliosis in the nucleus ambiguus in a patient with multiple system atrophy and vocal cord palsy. 
Figure 2 Complete section of the laryngeal branch removed at the lower border of the posterior cricoarytenoid muscle in a control patient. Transverse semithin sections stained with toluidine blue show two distinct patterns. Type 1 consists of two fascicles, one large and the other small, and type 2 of only one fascicle subdivided into two regions, one large and the other small, with a clear border in between as shown by two arrows. The same characteristics in fibre diameter histogram and transverse branch area allow the equating of the large and small fascicles in type 1 and the large and small regions in type 2. $M F=$ myelinated fibres; $A=$ area.

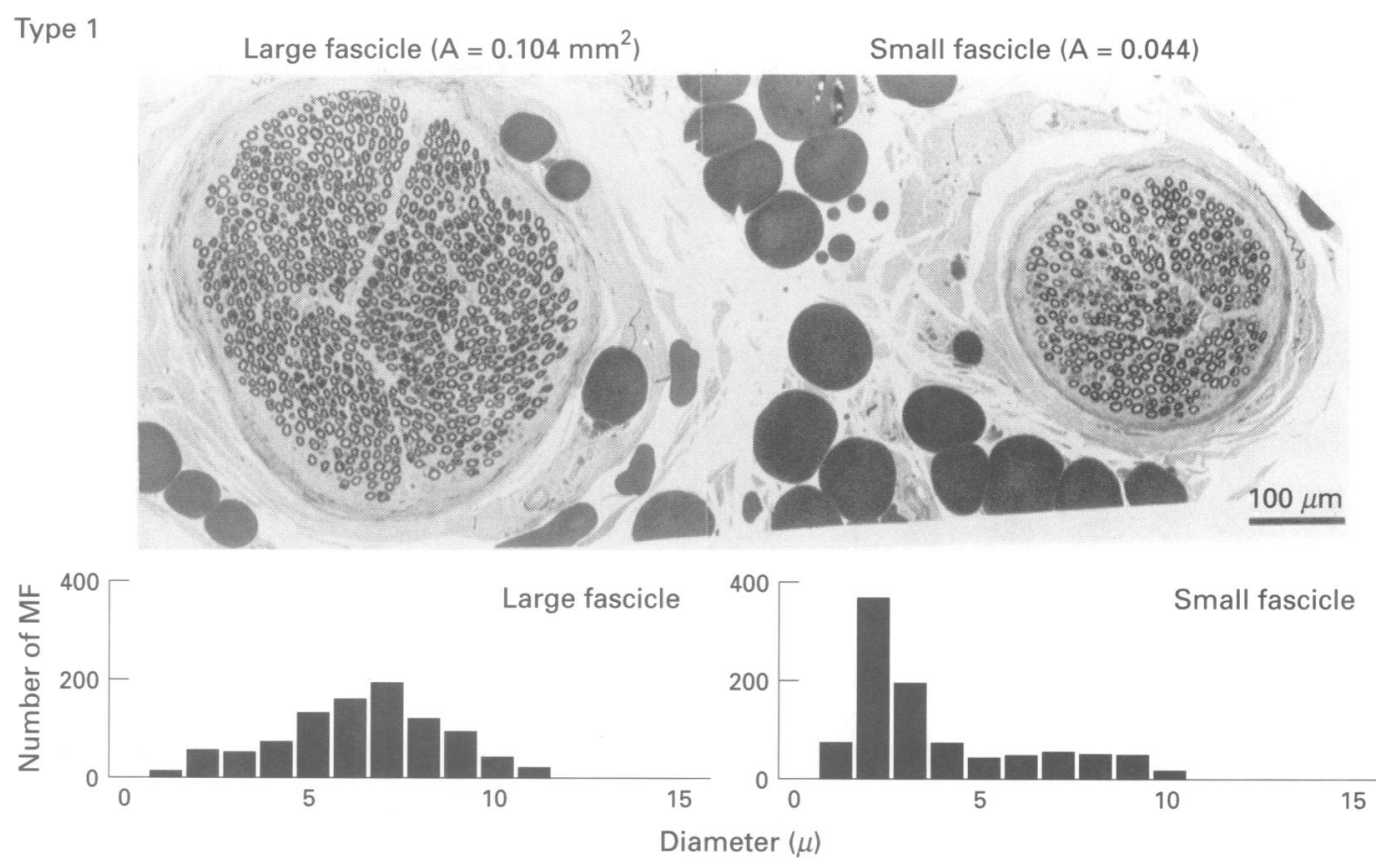

Type 2 serves the intrinsic laryngeal muscles, including the posterior cricoarytenoid muscles. ${ }^{5}$ The purpose of the present study was to further elucidate the innervation patterns of the posterior cricoarytenoid muscles and to establish characteristic abnormalities of the motor branches typically seen in multiple system atrophy. Specifically, we counted the number of myelinated nerve fibres in the laryngeal branch of the recurrent laryngeal nerve to construct histograms of the fibre diameter of its sensory and motor branches in patients with multiple system atrophy with and without vocal cord palsy.

\section{Materials and methods}

We obtained the laryngeal branches of the recurrent laryngeal nerve at necropsy from six patients with multiple system atrophy, four with and two without vocal cord palsy, and six patients without multiple system atrophy, who died of cerebral infarction or lung cancer. The patients with multiple system atrophy with and without vocal cord palsy were matched in age (mean 57.5 $v 55.5$ years) and duration of illness (mean 6.8 and 6.0 years). The control subjects had neither evidence of vocal cord malfunction such as stridor nor history of potential causes of neuropathy such as diabetes or alcoholism. The presence or absence of vocal cord palsy was confirmed by fibre laryngoscopy.

The posterior cricoarytenoid muscles were removed in five of the six patients with multiple system atrophy together with the other intrinsic laryngeal muscles-interarytenoid, lateral cricoarytenoid, and cricothyroid. Cryosections excised from these muscles were stained with haematoxylin and eosin and ATPase. The 
Figure 3 Diffuse, nontopical loss of myelinated nerve fibres was seen selectively in the large region. Arrows show borders between large and small regions. Compared with a patient without multiple system atrophy (A), there was only a mild change in the patients with multiple system atrophy without vocal cord palsy (B) and a notable change in those with vocal cord palsy (C).

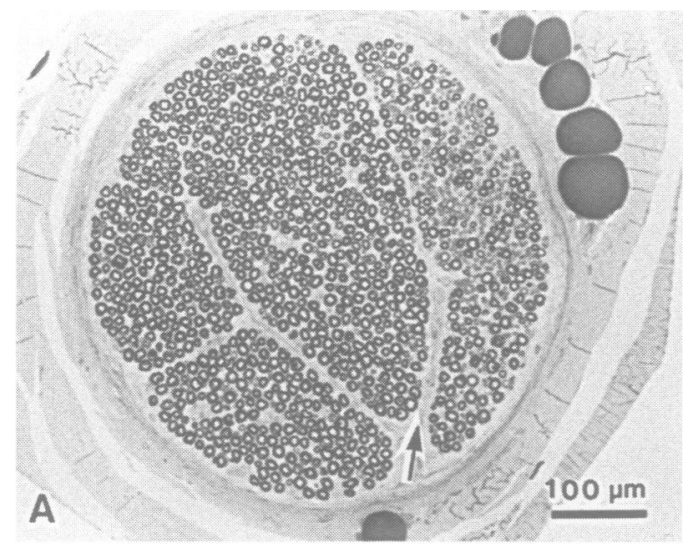

pH 7.4 for 12 hours and postfixation in $1 \%$ osmium tetroxide for three hours. Transverse semithin $(0.5 \mu \mathrm{m})$ sections were cut and stained with toluidine blue. For measurement of the transverse branch area $\left(\mathrm{mm}^{2}\right)$, using photomicrographs of $\times 180$ magnification we followed the outer edge of the branches with the cursor of the digitiser attachment on a programmed personal computer (NEC PC9801). Photomicrographs of $\times 1200$ were used to estimate myelinated nerve fibre density (number $/ \mathrm{mm}^{2}$ ) and the fibre diameter distribution of the myelinated fibres. For fibre diameter analysis, the lengths of the minor axes of the myelinated nerve fibres were measured with the digitiser of the computer to plot the fibre diameter distribution on a linear scale at $1.0 \mu \mathrm{m}$ intervals. The number of myelinated nerve fibres (number/branch) was calculated from the density and transverse branch area.

\section{Results}

In the patients with multiple system atrophy with vocal cord palsy, the posterior cricoarytenoid muscles were thin in gross appearance bilaterally, showing pronounced neurogenic changes (fig 1B). A clear grouped atrophy was seen in all, whereas fibre type grouping was more prominent in some than others, suggesting various degrees of reinnervation. By contrast, no abnormal histopathological changes were found in those without vocal cord palsy. The other intrinsic laryngeal muscles showed no detectable abnormalities in either group.

Examination of a complete section of the laryngeal branch removed at the lower border of the posterior cricoarytenoid muscles disclosed two distinct patterns: type 1 consisting of large and small fascicles (fig 2, top), and type 2 with one fascicle comprising large and small regions (fig 2 , bottom). At this level the section contained no other fascicles except for a fine and probably visceral nerve branch. In type 1, larger fascicles corresponded to the anterior motor branch innervating the posterior cricoarytenoid muscles, showing unimodal distribution of nerve fibres with a greater proportion of large diameter fibres. By contrast, smaller fascicles, representing the posterior sensory branch, had a greater percentage of small diameter fibres. In type 2 , the fibre diameter distribution and the transverse branch area in the large and small regions in each fascicle corresponded to the large and small fascicles in type 1 . These findings allowed us to equate the fascicles in type 1 to the regions in type 2, the larger ones representing motor branches, and the smaller ones sensory branches.

The patients with multiple system atrophy with vocal cord palsy had a significantly smaller transverse branch area for the motor branches than those without vocal cord palsy or the controls (fig 3 and table). ${ }^{1}$ Similarly, the patients with multiple system atrophy with vocal cord palsy had significantly fewer myelinated fibres for the motor branches-averaging only $33 \%$ of controls - than those without vocal cord palsy-averaging $66 \%$ (fig 3 and 
Number of myelinated nerve fibres and transverse branch area in multiple system atrophy (mean (SD, range)

\begin{tabular}{|c|c|c|c|}
\hline & $\begin{array}{l}M S A \text { with } V C P \\
(n=4)\end{array}$ & $\begin{array}{l}\text { MSA without VCP } \\
(n=2)\end{array}$ & $\begin{array}{l}\text { Controls } \\
(n=6)\end{array}$ \\
\hline \multicolumn{4}{|l|}{ Motor branch: } \\
\hline Number of myelinated fibres & $\begin{array}{l}295\left(85,{ }^{\star \star \star}+198-404\right) \\
(n=4)\end{array}$ & $\begin{array}{l}565(54, \star 526-603) \\
(\mathrm{n}=2)\end{array}$ & $\begin{array}{l}895(67,820-979) \\
(\mathrm{n}=6)\end{array}$ \\
\hline Transverse branch area $\left(\mathrm{mm}^{2}\right)$ & $\begin{array}{l}0.070\left(0.011,{ }^{\star \star}+† 0.055-0.080\right) \\
(\mathrm{n}=4)\end{array}$ & $\begin{array}{l}0 \cdot 114(0 \cdot 001,(\mathrm{NS}) 0 \cdot 114-0 \cdot 115) \\
(\mathrm{n}=2)\end{array}$ & $\begin{array}{l}0 \cdot 114(0.018,0.090-0 \cdot 138) \\
(\mathrm{n}=6)\end{array}$ \\
\hline \multicolumn{4}{|c|}{$(\mathrm{n}=2)$} \\
\hline & $\begin{array}{l}787(30,(N S) 766-808) \\
(n=2)\end{array}$ & $\begin{array}{l}485 \\
(n=1)\end{array}$ & $\begin{array}{l}753(238,570-1145) \\
(n=5)\end{array}$ \\
\hline Transverse branch area $\left(\mathrm{mm}^{2}\right)$ & $\begin{array}{l}0.069(0.021,(N S) 0.054-0.083) \\
(n=2)\end{array}$ & $\begin{array}{l}0.045 \\
(\mathrm{n}=1)\end{array}$ & $\begin{array}{l}0.045(0.008,0.032-0.050) \\
(n=5)\end{array}$ \\
\hline
\end{tabular}

${ }^{\star} \mathrm{P}<0.05 ;{ }^{\star \star} \mathrm{P}<0.005 ;{ }^{\star \star \star} \mathrm{P}<0.0001 v$ controls; $+\mathrm{P}<0.05 ; \mathrm{H} \mathrm{P}<0.01 v$ MSA without VCP; NS = non-significant $v$ controls. MSA $=$ multiple system atrophy; VCP, vocal cord palsy.

Figure 4 Fibre diameter histograms of the motor division of the laryngeal branch in patients with multiple system atrophy with and without vocal cord palsy, compared with controls. The small diameter fibres (diameter $<7 \mu \mathrm{m}$ ) were decreased in both groups, whereas the large diameter fibres (diameter $<8 \mu \mathrm{m}$ ) were lost only in those with vocal cord palsy; $M S A=$ multiple system atrophy; $V C P=$ vocal cord palsy.

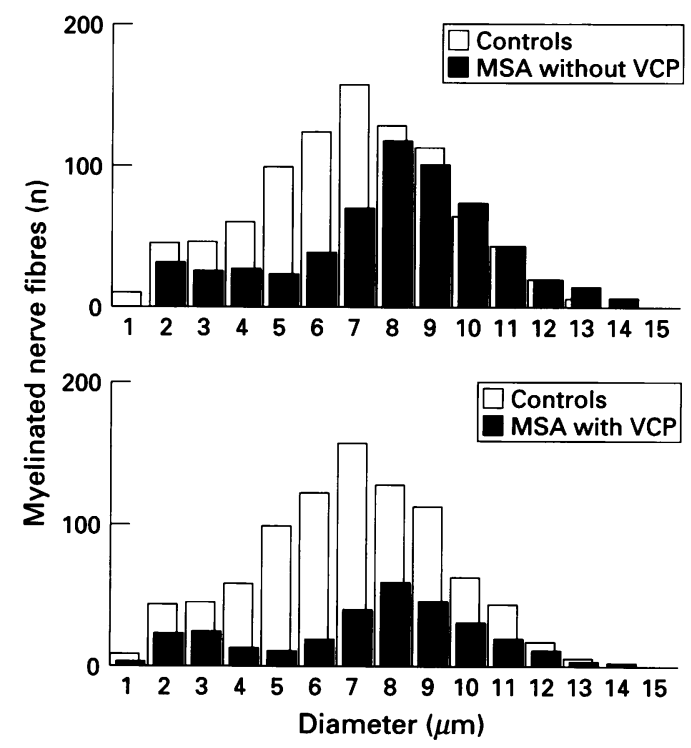

table). Both groups had significantly fewer myelinated fibres than the controls (table). By contrast, there were no significant differences in transverse branch area or number of myelinated fibres for the sensory branches between the patients with multiple system atrophy with or without vocal cord palsy and the controls.

Both large and small myelinated fibres were lost in the patients with multiple system atrophy with vocal cord palsy, whereas only the small diameter fibres were affected in those without vocal cord palsy (fig 4). The small myelinated fibres with diameters less than $7 \mu \mathrm{m}$ were decreased in those with vocal cord palsy and, to a lesser extent, also in those without vocal cord palsy. The large diameter fibres greater than $8 \mu \mathrm{m}$ were affected only in those with vocal cord palsy and preserved in those without vocal cord palsy.

\section{Discussion}

The motor and sensory fibres of the laryngeal branch of the recurrent laryngeal nerve analysed in the present study probably correspond to the two types of fascicles described by Hoffman and Schnitzlein, although in their report no quantitative data were presented regarding either large or small myelinated fibres. ${ }^{6}$ In the series reported by Schnitzlein et $a l$, the number of myelinated nerve fibres ranged from 1116 to 4096 (mean 2553) in the human left recurrent laryngeal nerve ${ }^{7}$ compared with our control values of 1516 to 2097 (mean 1699). The discrepancy between the two studies probably resulted from different sampling sites, ours being more distal.

By comparison with the controls, patients with multiple system atrophy had a decreased number of myelinated fibres in the motor but not in the sensory division of the laryngeal branch. The small myelinated nerve fibres were decreased in all patients with multiple system atrophy with additional loss of the large myelinated fibres only in those with vocal cord palsy. These findings imply that, in multiple system atrophy, the small diameter myelinated fibres are affected first before the loss of the large diameter fibres causing vocal cord palsy. Fibres with diameters larger than $8 \mu \mathrm{m}$ in the ventral roots are equated with the $\alpha$ motor axons. ${ }^{8}$ Although we examined the branches distal to the ventral roots, the large diameter fibres had similar characteristics to the $\alpha$ axons in the ventral roots. We speculate, therefore, that these large diameter fibres correspond to the $\alpha$ axons that innervate the intrinsic laryngeal muscles whereas the small diameter fibres represent other components, such as $\gamma$ axons. The involvement of the $\alpha$ axons of the recurrent laryngeal nerve, rather than the small myelinated axons, results in neurogenic atrophy of the posterior cricoarytenoid muscles, which in turn leads to vocal cord palsy.

Whether multiple system atrophy characteristically affects the neurons of the nucleus ambiguus is a matter for controversy. Lapresle and Annabi reported loss of neurons in the lower two thirds of the nucleus ambiguus in serial sections of the medulla. ${ }^{9}$ Bannister et al found no loss of neurons in their samples despite neurogenic atrophy of the posterior cricoarytenoid muscles. ${ }^{2}$ Such discrepancy stems in part from technical difficulties experienced in morphometric studies of this nucleus. Serial sections made over the entire length of the nucleus are required to avoid sampling error caused by the uneven distribution of neurons. ${ }^{1011}$ Moreover, identification of the motor neurons is not always easy. In our series, all the tissue samples of the nucleus ambiguus obtained from patients with multiple system atrophy showed gliosis (fig 1C). In an earlier study based on observations of entire serial sections taken from the medullas, Isozaki reported a loss of the neurons in the caudal two thirds of this nucleus in two patients with multiple system atrophy with vocal cord palsy (patients 2 and 3 with multiple system atrophy in our series). ${ }^{11} \mathrm{~A}$ decrease in 
the number of myelinated fibres seen in the present study also suggests a loss of the motor neurons that innervate the intrinsic laryngeal muscles. Thus although further proof is necessary before definite conclusions can be drawn, the currently available data implies neuronopathy involving the nucleus ambiguus as a cause of vocal cord palsy in some patients with multiple system atrophy.

The laryngeal branch of the recurrent laryngeal nerve supplies not only the posterior cricoarytenoid muscles but also other intrinsic laryngeal muscles - which include the interarytenoid, lateral cricoarytenoid, and cricothyroid muscles. Neurogenic changes were, however, confined to the posterior cricoarytenoid muscles in our patients, in agreement with results in previous reports. ${ }^{29}$ These findings imply that the bundle of nerve fibres destined to innervate posterior cricoarytenoid muscles is selectively affected in multiple system atrophy. None the less, we found diffuse rather than topical loss of myelinated nerve fibres in this part of the motor branches subserving the laryngeal branch of the recurrent laryngeal nerve (fig 3). To rule out selectivity at the neural level, it is necessary to establish the innervation pattern of the intrinsic laryngeal muscles more clearly. At this level, the nerve fibres innervating different muscles may be mingled without forming separate bundles. Histological studies of the recurrent laryngeal nerve in cats using the horseradish peroxidase tracer technique disclosed no grouping of the nerve fibres that subserved the laryngeal abductor and adductor muscles. ${ }^{12}$ Results of partial ligation of equine recurrent laryngeal nerve also suggested mingling of the myelinated nerve fibres for the abductors and adductors within the nerve. ${ }^{13}$ Under this circumstance, it is unlikely that focal lesions of the recurrent laryngeal nerve result in selective denervation of individual laryngeal muscles.
Alternatively, it is possible that the posterior cricoarytenoid muscles are more vulnerable than the other laryngeal muscles to motor neuropathy affecting the laryngeal branch of the recurrent laryngeal nerve as a whole. Regardless of the underlying aetiology, the loss of the large myelinated fibres in the laryngeal branch of the recurrent laryngeal nerve results in relatively selective atrophy of posterior cricoarytenoid muscles in advanced cases of multiple system atrophy with vocal cord palsy.

1 Williams A, Hanson D, Calne DB. Vocal cord paralysis in the Shy-Drager syndrome. 7 Neurol Neurosurg Psychiatry 1979;42:151-3.

2 Bannister R, Gibson W, Michaels L, Oppenheimer DR. Laryngeal abductor paralysis in multiple system atrophy. A report on three necropsied cases, with observations on the laryngeal muscles and the nuclei ambigui. Brain 1981;104:351-68.

3 Munschauer FE, Loh L, Bannister R, Newsom-Davis J. Abnormal respiration and sudden death during sleep in multiple system atrophy with autonomic failure. Neurology 1990;40:677-9.

4 Guindi GM, Bannister R, Gibson WP, Payne JK. Laryngeal electromyography in multiple system atrophy Laryngeal electromyography in multiple system atrophy
with autonomic failure. $f$ Neurol Neurosurg Psychiatry with autonomic

5 Steinberg JL, Khane GJ, Fernandes CMC, Nel JP. Anatomy of the recurrent laryngeal nerve: a redescription. F Laryngol Otol 1986;100:919-27.

6 Hoffman $\mathrm{HH}$, Schnitzlein HN. The numbers of nerve fibres in the vagus nerve of man. Anat Rec 1961;139: 429-35.

7 Schnizlein HN, Rowe LC, Hoffman HH. The myelinated component of the vagus nerves in man. Anat Rec 1958; 131:649-67.

8 Kawamura Y, Okazaki H, O'Brien PC, Dyck PJ. Lumbar motoneurons of man. I: Number and diameter histogram of alpha and gamma axons of ventral root. $\mathcal{F}$ Neuropathol Exp Neurol 1977;36:853-60.

9 Lapresle J, Annabi A. Olivopontocerebellar atrophy with velopharyngolaryngeal paralysis: a contribution to the velopharyngolaryngeal paralysis: a contribution to the Nomatotopy of the nucle

10 Tomasch J, Ebnessajjade D. The human nucleus ambiguus. A quantitative study. Anat Res 1961;3: 247-52.

11 Isozaki E. Clinicopathological, electromyographical, and phoniatric studies on bilateral vocal cord paralysis in

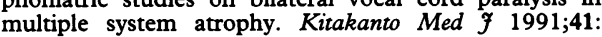
389-409. (In Japanese.)

12 Malmgren LT, Lyon MJ, Gacek RR. Localization of abductor and adductor fibres in the kitten recurrent laryngeal nerve: use of a variation in the horseradish peroxidase tracer technique. Exp Neurol 1977;55:187-98.

13 Dyer KR, Duncan ID. The intraneural distribution of myelinated fibres in the equine recurrent laryngeal nerve. Brain 1987;110:1531-43. 